\title{
Mesenchymal stem cells have significant anti-infective effect on methicillin-resistant Staphylococcus epidermidis vascular graft infections
}

\author{
Mezenkimal kök hücreler metisiline dirençli Stafilokokus epidermidis vasküler greft enfeksiyonları \\ üzerinde önemli anti-enfektif etkiye sahiptir
}

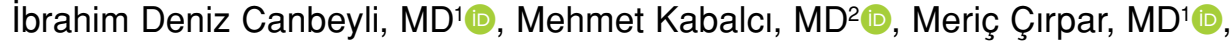 \\ Meral Tiryaki, $\mathrm{PhD}^{3}$ iD, Birhan Oktaş, $\mathrm{MD}^{1}$ (B) \\ 1Department of Orthopedics and Traumatology, Kırıkkale University, School of Medicine, Kırıkkale, Turkey \\ 2Department of Cardiovascular Surgery, Kırıkkale University, School of Medicine, Kırıkkale, Turkey \\ ${ }^{3}$ Department of Pathology, Dışkapı Yıldırım Beyazıt Training and Research Hospital, Ankara, Turkey
}

\begin{abstract}
Objectives: This study aims to evaluate the effects of mesenchymal stem cell (MSC) implantation on vascular graft infections caused by methicillinresistant Staphylococcus epidermidis (MRSE) and compare with antibiotic treatment.

Materials and methods: Healthy adult 56 Wistar rats (age, over 5 months; weighing, 300-350 g) were divided into eight groups. Group 1 was defined as the control group and group 2 was defined as the infected control group. Groups 3 and 4 were defined as Dacron grafted and MRSE infected groups, treated with tigecycline and MSCs, respectively. Groups 5 and 6 were performed polytetrafluoroethylene (PTFE) graft and infected with MRSE. These groups were also administered tigecycline and MSC treatment, respectively. Groups 7 and 8 were infected with MRSE without graft administration and were also performed tigecycline and MSC treatment, respectively. Grafts and soft tissue specimens were collected at 13 days postoperatively. Colony counts of peri-graft tissue were performed. All samples were evaluated by enzyme-linked immunosorbent assay (ELISA) for the markers that determine stem cell activity.

Results: The overall success of the treatments was assessed by the number of rats with MRSE recurrence, regardless of graft used. The difference between the untreated group 2 , tigecycline groups $(3,5$ and 7$)$ and MSCs groups (4, 6 and 8 ) were statistically significant. Success of MSC and tigecycline treatments was similar in Dacron, PTFE, and non-grafted groups. There was a resistance of MRSE infection in Dacron groups to MSC and tigecycline treatments. This was considered to be indicative of the susceptibility of the Dacron grafts to infection. However, there was no significant difference between group 2 and Dacron groups in terms of bacterial colonization. ELISA results were significant in three cytokines.

Conclusion: Mesenchymal stem cells can be considered as an alternative treatment option on its own or part of a combination therapy for control of vascular graft infections.
\end{abstract}

Keywords: Dacron, mesenchymal stem cells, polytetrafluoroethylene, tigecycline, vascular graft infections.

\section{ÖZ}

Amaç: Bu çalışmada, mezenkimal kök hücre $(\mathrm{MKH})$ implantasyonunun metisiline dirençli Stafilokokus epidermidis (MDSE)'in neden olduğu vasküler greft enfeksiyonları üzerindeki etkileri değerlendirildi ve antibiyotik tedavisi ile karşılaştırıldı.

Gereç ve yöntemler: Sağlıklı erişkin 56 Wistar sıçanı (yaş, 5 ay üzeri; ağırlık, 300-350 g) sekiz gruba ayrildı. Grup 1 kontrol grubu, grup 2 enfekte kontrol grubu olarak tanımlandı. Grup 3 ve 4 Dakron grefti uygulanan, MDSE ile enfekte olan, sırasiyla tigesiklin ve $\mathrm{MKH}$ tedavisi uygulanan gruplar olarak tanımlandı. Grup 5 ve 6 politetrafloroetilen (PTFE) greft uygulanarak MDSE ile enfekte edildi. Bu gruplara da sirasıyla tigesiklin ve MKH tedavisi uygulandı. Grup 7 ve 8 greft uygulanmadan, MDSE ile enfekte edildi ve sirasılya bu gruplara da tigesiklin ve MKH tedavisi uyguland. Greftler ve yumuşak doku örnekleri ameliyattan 13 gün sonra alındı. Greft çevresi dokuda koloni sayımı yapıldı. Tüm numuneler, kök hücre aktivitesini gösteren belirteçler açısından enzime bağ 1 bağışıklık deneyi (ELISA) ile değerlendirildi.

Bulgular: Tedavilerin genel başarısı, kullanılan greftten bağımsız olarak, MDSE rekürrensi olan sıçanların sayısı ile değerlendirildi. Tedavi edilmeyen grup 2, tigesiklin grupları (3,5 ve 7) ile MKH grupları (4, 6 ve 8$)$ arasındaki farklılık istatistiksel olarak anlamlıydı. Mezenkimal kök hücre ve tigesiklin tedavilerinin başarısı Dakron, PTFE ve greft uygulanmayan gruplarda benzerdi. Dakron gruplarında MDSE enfeksiyonunun hem MKH hem de tigesiklin tedavisine karşı bir direnci vardı. Bu, Dakron greftlerinin enfeksiyona duyarlılı̆ı̆ının bir göstergesi olarak kabul edildi. Ancak, grup 2 ve Dakron grupları arasında bakteriyel kolonizasyon açısından anlamlı farkl11ık yoktu. ELISA sonuçları üç sitokinde anlamlıydı.

Sonuç: Mezenkimal kök hücreler, tek başına alternatif bir tedavi seçeneği veya vasküler greft enfeksiyonlarının kontrolü için kombine terapinin bir parçası olarak kabul edilebilir.

Anahtar sözcükler: Dakron, mezenkimal kök hücreler, politetrafloroetilen, tigesiklin, vasküler greft enfeksiyonları.

Received: March 26, 2019 Accepted: June 25, 2019 Published online: October 24, 2019

Correspondence: Ibrahim Deniz Canbeyli, MD. Kırıkale Üniversitesi Tıp Fakültesi Ortopedi ve Travmatoloji Anabilim Dalı, 71450 Yahșihan, Kırıkkale, Turkey. Tel: +90 $532-2214147$ e-mail: denizcanbeyli@gmail.com 
The incidence of vascular injury following extremity trauma varies widely according to geographic location (rural, urban), population (civilian, military), and mechanisms of injury (penetrant, blunt).[1] Complex extremity trauma, involving both arterial and skeletal injuries is a clinical challenge. Orthopedic trauma surgeons are more likely to face this combination of injury than vascular and trauma surgeons since most of the limb arterial injuries are associated with skeletal trauma. ${ }^{[2]}$ Particularly Gustilo-Anderson grade 3C fractures, which are associated with severe soft tissue loss and vascular injury, usually require vascular repair. In these complex injuries, the frequency of deep infections and need for major limb amputations are high and multiple surgeries over long periods of time are often required to obtain healing. ${ }^{[3]}$ Despite all precautions and antibiotic use, death rates due to vascular graft infections $(15-75 \%)^{[1]}$ and limb amputations $(70 \%)$ are still high. ${ }^{[4,5]}$ On the other hand, delay in diagnosis and treatment of vascular injury, particularly when complicated by infection and graft thrombosis, may raise the rates of extremity amputations. ${ }^{[6]}$ Prosthetic vascular graft infections occur in up to $6 \%$ of patients having bypass distal to inguinal level at the lower extremity. ${ }^{[4]}$ Against alternative treatment approaches as in situ graft replacement ${ }^{[7]}$ and graft retention with thorough debridement, ${ }^{[8]}$ removal of the graft followed by extraanatomic bypass revascularization is the traditional treatment method for vascular graft infections. Methicillin-resistant Staphylococcus epidermidis (MRSE) is the common cause of these infections, ${ }^{[9,10]}$ which usually does not respond to antibiotherapy, requiring graft excision. ${ }^{[11]}$

Mesenchymal stem cells (MSCs) have immunomodulatory and anti-inflammatory effect achieved by change in $\mathrm{T}$ helper subtypes, regulation of the activity of macrophages and changing proliferation, differentiation and immunsecretion characteristics of B cells. ${ }^{[12,13]}$ They also show direct antibacterial effect achieved by secretion of antibacterial peptides and augmentation of phagocytosis. ${ }^{[14]}$ The efficacy of treatment with allogeneic and autogenic MSC transplantation has been demonstrated on myocardial infarction, ${ }^{[15]}$ diabetes, ${ }^{[16]}$ graft versus host disease, ${ }^{[17]}$ acute infectious lung injury, ${ }^{[18]}$ sepsis and organ dysfunction, ${ }^{[19]}$ staphylococcus toxic shock syndrome, ${ }^{[20]}$ and peritonitis. ${ }^{[21]}$ Thus, the anti-infectious effect of MSCs may be studied on other clinically resistive forms of infection.

In this study, we hypothesize that MSCs can be an effective treatment option in vascular graft infections due to their direct anti-microbial and immunomodulating effects in patients who need treatment of vascular graft infections, particularly who are unable to use antibiotics or require dose adjustment due to the side effects. Thus, in this study, we aimed to evaluate the effects of MSCs implantation on vascular graft infections caused by MRSE and compare with antibiotic treatment.

\section{MATERIALS AND METHODS}

This study was conducted at Kirıkkale University Medical Faculty between 10 August 2016 and 21 December 2017 with the approval of the Local Animal Experiments Ethics Committee dated 10.06.2016 and numbered 16/61. All animals were maintained in accordance with the principles of animal care developed by the National Academy of Sciences (Guide for the Care and Use of Laboratory Animals). We conducted a power analysis to determine the size of the study group prior to the ethics committee application. During the entire study, rats were examined under veterinary supervision, fed a standard diet without water deprivation, at $22 \pm 1.9^{\circ} \mathrm{C}$ room temperature and $52 \pm 6 \%$ relative humidity, in the Experimental Animal Research Laboratory. ${ }^{[22]}$

Healthy adult 56 Wistar rats (age, over 5 months; weighing, 300-350 g) were randomly divided into eight groups, each group consisting of seven rats. Group 1 was defined as the control group. No graft was implanted in these rats. They were uncontaminated and did not receive antibiotic treatment or MSCs implantation. Group 2 was defined as the infected control group. These rats were not grafted with Dacron or polytetrafluoroethylene (PTFE). They were locally infected with MRSE and not treated with antibiotics or MSCs. In group 3, Dacron graft was implanted into rats, they were locally infected with MRSE and treated with intraperitoneal tigecycline $10 \mathrm{mg} / \mathrm{kg}$ for 10 days twice daily. Dacron grafting was also performed for group 4 rats which were again locally infected with MRSE and treated with single dose local implantation of $2 \times 10^{6}$ MSCs. Group 5 rats had PTFE graft implantation infected with MRSE and treated with intraperitoneal tigecycline $10 \mathrm{mg} / \mathrm{kg}$ for 10 days twice daily. Group 6 rats also received PTFE grafting, locally infected with MRSE and treated with single dose local administration of $2 \times 10^{6}$ MSCs. Group 7 rats were the non-grafted, infected rats with MRSE and they received intraperitoneal tigecycline $10 \mathrm{mg} / \mathrm{kg}$ for 10 days twice daily. Group 8 rats were also non-grafted, locally infected with MRSE and treated with single dose local administration of $2 \times 10^{6}$ MSCs. 
Methicillin-resistant Staphylococcus epidermidis was isolated from a 72-year-old male patient with osteomyelitis in 2016. Samples from the infected area were obtained and taken for routine bacteriological studies at the Department of Microbiology of the Veterinary Medicine at Kırıkkale University. Clinical isoforms were determined by gram staining, catalase reaction, tube coagulase test and API-Staph test (bioMérieux, Lyon, France). Methicillin susceptibility was determined using the Kirby Bauer Disk Diffusion method. ${ }^{[23]}$ Susceptibility of MRSE strains to antimicrobial effect of tigecycline was determined according to Clinical and Laboratory Standards Institute procedures and Kirby Bauer Disk Diffusion method. Tigecycline (TYGACIL, Pfizer ${ }^{\circledR}$, NY, USA) dose $(10 \mathrm{mg} / \mathrm{kg})$ was adjusted according to the dosage regimen found effective in animal studies. ${ }^{[24]}$

Diagnosis of infection was confirmed by gram staining and culture of samples obtained in group 2 infected control group of rats. Organisms were quantified as the number of colony-forming units (CFUs) per container.

Fast thaw technique was used ${ }^{[25]}$ where cells were thawed by transferring warmed media to a frozen tube quickly and transferring to a reaction tube. The tissue was rinsed to remove blood cells and then mechanically separated into small pieces that were no longer than a few millimeters. ${ }^{[26]}$

Rat adipose cells were taken from a rat and used to prepare Rat MSCs at Yildirim Beyazit University Stem Cell Laboratory. Mesenchymal stem cells were isolated from rat inguinal area and subcutaneous flank adipose tissue. Explant culture technique was used to isolate the MSCs. Explant culture is one of the earliest techniques of cell isolation and in vitro cell culture. Small piece tissues are placed in culture dishes and cells migrate out to adhere to the culture surface; no enzyme is used in this technique. ${ }^{[27,28]}$ Cell counts and survival were performed using the Countess $^{\circledR}$ Automated Cell Counter (Thermo Fisher Scientific, Waltham, USA).

All animals were sedated intramuscularly $(0.75 \mathrm{~mL} / \mathrm{kg})$ with $2: 1$ mixed ketamine hydrochloride (100 mg/mL; Pfizer, Luleburgaz, Turkey) and xylazine hydrochloride $(20 \mathrm{mg} / \mathrm{mL}$; Bayer AG, Leverkusen, Germany). The fur on the back of each rat was shaved and disinfected with a $10 \%$ povidone-iodine solution. A subcutaneous pocket was made with $1 \mathrm{~cm}$ cuts in the middle of the back (Figure 1). ${ }^{[29]} 1 \mathrm{~cm}^{2}$, woven, gelatin impregnated Dacron grafts (Gelweave, Sulzer Vascutek Ltd., Scotland, United Kingdom) and $1 \mathrm{~cm}^{2}$ PTFE grafts (Gore-Tex; W.L. Gore \& Associates Inc.,
Newark, USA) were used. Twenty-four hours later, sterile saline solution $(1 \mathrm{~mL})$ equal to MRSE at $2 \times 10^{6}$ CFU per $\mathrm{mL}$ was inoculated onto the surface of the grafts with a tuberculin syringe. After 48 hours, sterile saline solution ( $1 \mathrm{~mL})$ equivalent to MRSE at $2 \times 10^{6}$ CFU per $\mathrm{mL}$ was inoculated onto the surfaces of groups 2, 7 and 8 grafts with a tuberculin syringe. Animals were individually housed in cages and checked daily for wound healing. All grafts were implanted on the first day. On the second day, the infection agent (MRSE) was injected into the graft site. The anti-infective therapy was started on the third day. As advised by Goessens et al., ${ }^{[2]} 10$ days after the anti-infective treatment, all grafts were taken under sterile conditions on the $13^{\text {th }}$ day of graft implantation. After this process, all animals were sacrificed.

Mesenchymal stem cells express various growth factors (e.g., vascular endothelial growth factor [VEGF]; hepatocyte growth factor; insulin-like growth factor-1; fibroblast growth factor [FGF]; keratinocyte growth factor; transforming growth factor-beta [TGF- $\beta]$ ) and anti-inflammatory cytokines (e.g., prostaglandin E2). ${ }^{[30]}$ Proinflammatory cytokines, particularly interleukin 1 (IL-1) and tumor necrosis factor alpha (TNF- $\beta$ ) upregulated during the inflammatory process. ${ }^{[3]}$ The standard enzyme-linked immunosorbent assay method was used for analyzing quantification of FGF, TGF- $\beta 1$, IL-1 alpha (IL-1 $\alpha$ ), VEGF, TNF- $\alpha$, plateletderived growth factor (PDGF) and caspase 3 (CASP3) cytokines $^{[32]}$ to evaluate the effect of MSCs in the infected tissue samples.

\section{Statistical analysis}

Statistical analyses were performed using the IBM SPSS version 24.0 (IBM Corp., Armonk, NY, USA) software. The variables were investigated using

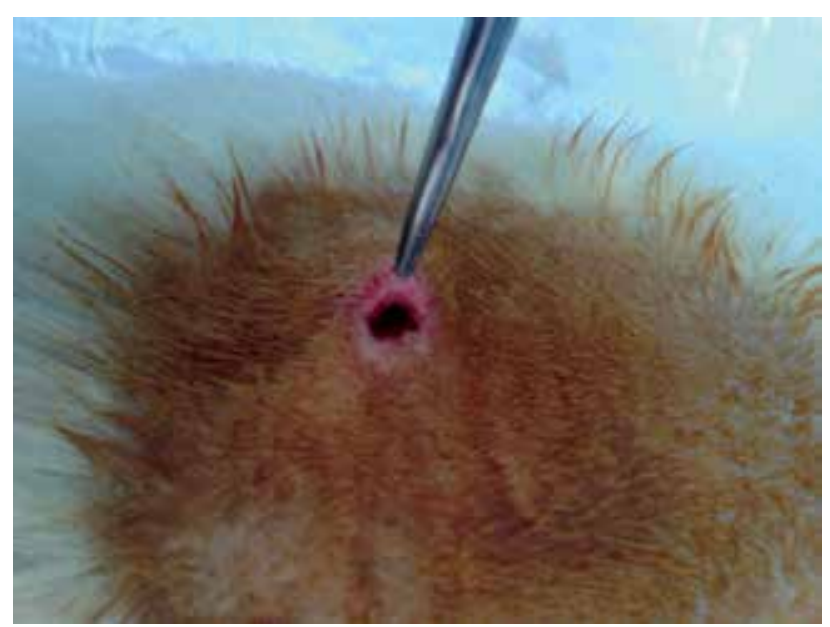

Figure 1. Subcutaneous pocket on dorsal of rat. 
visual (histograms, probability plots) and analytical methods (Kolmogorov-Smirnov/Shapiro-Wilk's test) to determine whether they are normally distributed. Descriptive statistics were presented as median (first quantile-third quantile). Normally distributed data were analyzed by one-way analysis of variance (ANOVA) followed by Kruskal Wallis. Data were shown as mean \pm standard deviation of absolute number. The Mann-Whitney $U$ test was used to compare the success of treatment between the groups. Bonferroni correction was used to adjust for multiple comparisons. The chi-square test or Fisher's exact test, where appropriate, was used to compare groups in terms of the number of rats in which reproduction was detected. A two tailed $p$ value less than 0.05 was considered to show a statistically significant result.

\section{RESULTS}

There was no animal that died for any reason during the study. In addition, local effects, anorexia, nausea, vomiting, diarrhea or behavioral disturbances were not observed in any group.

There was a significant difference in terms of bacterial colonization between group 1 (control group) and group 2 (infected control) $(\mathrm{p}=0.004)$. No treatment was applied to groups 1 and 2. In groups 3 and 4 , a small number of bacterial colonization was observed irrespective of treatment. However, the difference between groups 3 and 4 was not statistically significant. There was no bacterial recurrence occurred in groups 5, 6, 7 and 8. Bacterial colonization was not observed in MSCs-treated groups 6 and 8, whereas in group 4, another MSCstreated group, reproductive activity was detected. In groups 5 and 7 treated with tigecycline, there was no bacterial colonization, whereas, in group 3 , which was also treated with tigecycline, bacterial colonization was found to be minimal. The grafts placed in the tissues, the cultivated agents and the quantitative culture results are given in Table I.

The numbers of colonization according to grafts and treatments are given in Table II as mean, median $25 \%$ and median $75 \%$. There was a statistically significant difference between groups 1 and 2 in terms of colony numbers $(p<0.001)$. Both successes of groups 3 and 4 were statistically significant when compared with group $2(\mathrm{p}<0.001, \mathrm{p}<0.001)$. The treatment success was similar in groups 3 and $4(p=0.004)$. There was no statistically significant difference in terms of treatment success between the PTFE grafted groups $(\mathrm{p}=0.91)$. Groups 3, 5 and 7, treated with tigecycline, were compared in terms of colony counts. In groups 5 and 7, tigecycline treatment was more successful than group $3(\mathrm{p}<0.001)$. Groups 4,6 and 8, treated

TABLE I

Infection agents, treatments and reproductive outcomes

\begin{tabular}{lccccc}
\hline & Type of graft & MRSE & Tigecycline & MSCs & Counts of reproduction \\
\hline Group 1 & Non & - & - & - & $0^{*}$ \\
Group 2 & Non & $2 \times 10^{6}$ & - & - & $9.6 \times 10^{7} \pm 0.4 \times 10^{7}$ \\
Group 3 & Dacron & $2 \times 10^{6}$ & $2 \times 1$ (10 days) & - & $1.1 \times 10^{2} \pm 0.3 \times 10^{2}$ \\
Group 4 & Dacron & $2 \times 10^{6}$ & - & Single dose MSC & $0.8 \times 10^{2} \pm 0.1 \times 10^{2}$ \\
Group 5 & PTFE & $2 \times 10^{6}$ & $2 \times 1(10$ days) & - & $0^{*}$ \\
Group 6 & PTFE & $2 \times 10^{6}$ & - & Single dose MSC & $0^{*}$ \\
Group 7 & Non & $2 \times 10^{6}$ & $2 \times 1$ (10 days) & - & $0^{*}$ \\
Group 8 & Non & $2 \times 10^{6}$ & - & Single dose MSC & $0^{*}$ \\
\hline MRSE: Methicillin-resistant Staphylococcus epidermidis; MSCs: Mesenchymal stem cells: PTFE. Polytetrafluoroethylene; *No reproduction.
\end{tabular}

MRSE: Methicillin-resistant Staphylococcus epidermidis; MSCs: Mesenchymal stem cells; PTFE: Polytetrafluoroethylene; * No reproduction.

TABLE II

Statistical comparison of rats with respect to treatments according to graft use

\begin{tabular}{lcccc}
\hline & No-antibiotic & Tigecycline & MSCs & $p$ \\
\hline Non-grafted & $9.6(5.3-8.2) \times 10^{7}$ & $0(0-0)$ & $0(0-0)$ & $<0.001^{*}$ \\
Dacron grafted & - & $81(73-86)$ & $109(93-121)$ & $=0.004^{\star *}$ \\
Polytetrafluoroethylene grafted & - & $0(0-0)$ & $0(0-0)$ & $=0.91^{* *}$ \\
\hline
\end{tabular}

MSCs: Mesenchymal stem cells; Kruskal Wallis test median (25\%-75\%) $\leq 0.05$; ${ }^{*}$ Difference between treatments in non-grafted groups; ${ }^{\star *}$ Difference between treatments in groups with Dacron grafts; ${ }^{* \star}$ Difference between treatments in polytetrafluoroethylene grafted groups. 
TABLE III

Results of descriptive analysis

\begin{tabular}{|c|c|c|}
\hline & Mean \pm SD & Min-Max \\
\hline \multicolumn{3}{|l|}{ FGF } \\
\hline Control & $211.24 \pm 60.58$ & $163.43-316.90$ \\
\hline Infected control & $243.68 \pm 113.09$ & $11.53-370.14$ \\
\hline Dacron+TIG & $214.74 \pm 88.04$ & $71.04-321.60$ \\
\hline Dacron+MSCs & $174.77 \pm 42.18$ & $96.09-224.51$ \\
\hline PTFE+TIG & $292.96 \pm 121.29$ & $105.49-445.31$ \\
\hline PTFE+MSCs & $181.91 \pm 53.79$ & $116.45-251.13$ \\
\hline Non-grafted+TIG & $258.51 \pm 140.61$ & $105.49-445.31$ \\
\hline Non-grafted+MSCs & $175.58 \pm 72.29$ & $116.45-308.40$ \\
\hline \multicolumn{3}{|l|}{ TGF- $\beta 1$} \\
\hline Control & $61.83 \pm 20.75$ & $18.59-76.52$ \\
\hline Infected control & $71.29 \pm 32.63$ & $42.98-131.40$ \\
\hline Dacron+TIG & $54.74 \pm 23.25$ & $18.59-91.77$ \\
\hline Dacron+MSCs & $84.79 \pm 48.45$ & 39.93-164.94 \\
\hline PTFE+TIG & $66.06 \pm 17.67$ & $46.03-91.77$ \\
\hline PTFE+MSCs & $84.79 \pm 47.71$ & $42.98-177.14$ \\
\hline Non-grafted+TIG & $80.00 \pm 15.38$ & $55.18-97.86$ \\
\hline Non-grafted+MSCs & $74.77 \pm 27.98$ & $42.98-125.30$ \\
\hline \multicolumn{3}{|l|}{ IL-1 $1 \alpha$} \\
\hline Control & $113.39 \pm 34.17$ & $84.85-163.40$ \\
\hline Infected control & $33.01 \pm 16.54$ & $16.12-66.67$ \\
\hline Dacron+TIG & $71.53 \pm 35.13$ & $24.90-124.13$ \\
\hline Dacron+MSCs & $66.53 \pm 50.39$ & $22.66-140.49$ \\
\hline PTFE+TIG & $97.44 \pm 45.97$ & 61.94-166.67 \\
\hline PTFE+MSCs & $148.77 \pm 56.41$ & $92.30-251.77$ \\
\hline Non-grafted+TIG & $132.88 \pm 46.38$ & $48.85-189.59$ \\
\hline Non-grafted+MSCs & $120.80 \pm 70.99$ & $37.58-251.77$ \\
\hline \multicolumn{3}{|l|}{ VEGF } \\
\hline Control & $235.21 \pm 64.04$ & $154.41-359.46$ \\
\hline Infected control & $358.33 \pm 102.44$ & $235.55-493.40$ \\
\hline Dacron+TIG & $278.16 \pm 111.85$ & $118.34-440.12$ \\
\hline Dacron+MSCs & $374.36 \pm 111.96$ & $254.72-593.55$ \\
\hline PTFE+TIG & $485.10 \pm 129.00$ & $365.38-676.66$ \\
\hline PTFE+MSCs & $335.94 \pm 118.73$ & $218.50-580.77$ \\
\hline Non-grafted+TIG & $445.88 \pm 114.09$ & $342.10-676.66$ \\
\hline Non-grafted+MSCs & $292.01 \pm 54.02$ & $231.28-365.07$ \\
\hline \multicolumn{3}{|l|}{ TNF- $\alpha$} \\
\hline Control & $78.33 \pm 30.64$ & $48.71-135.97$ \\
\hline Infected control & $222.30 \pm 231.40$ & $40.77-730.95$ \\
\hline Dacron+TIG & $178.35 \pm 50.20$ & $124.91-256.42$ \\
\hline Dacron+MSCs & $98.54 \pm 35.99$ & $46.98-151.84$ \\
\hline PTFE+TIG & $197.36 \pm 46.40$ & $142.17-270.83$ \\
\hline PTFE+MSCs & $155.23 \pm 63.69$ & $96.31-245.36$ \\
\hline Non-grafted+TIG & $169.28 \pm 65.03$ & $72.51-270.83$ \\
\hline Non-grafted+MSCs & $182.48 \pm 183.88$ & $80.77-580.22$ \\
\hline \multicolumn{3}{|l|}{ PDGF } \\
\hline Control & $5.72 \pm .89$ & $4.31-6.79$ \\
\hline Infected control & $6.14 \pm .60$ & $5.22-6.89$ \\
\hline Dacron+TIG & $5.34 \pm 1.78$ & $2.42-6.99$ \\
\hline Dacron+MSCs & $5.57 \pm .86$ & $3.89-6.47$ \\
\hline PTFE+TIG & $6.76 \pm .84$ & $5.68-8.33$ \\
\hline PTFE+MSCs & $6.09 \pm .77$ & $4.79-6.78$ \\
\hline Non-grafted+TIG & $6.59 \pm 1.14$ & $4.68-8.33$ \\
\hline Non-grafted+MSCs & $5.74 \pm 1.23$ & $3.99-7.25$ \\
\hline
\end{tabular}

TABLE III

Continued

\begin{tabular}{lcc}
\hline & Mean \pm SD & Min-Max \\
\hline CASP3 & & \\
Control & $10.73 \pm 1.64$ & $8.26-12.70$ \\
Infected control & $11.95 \pm .87$ & $10.69-13.35$ \\
Dacron+TIG & $9.38 \pm 2.01$ & $6.71-12.17$ \\
Dacron+MSCs & $12.24 \pm 1.29$ & $10.59-14.73$ \\
PTFE+TIG & $13.19 \pm 1.10$ & $11.44-14.61$ \\
PTFE+MSCs & $12.89 \pm .66$ & $11.73-13.73$ \\
Non-grafted+TIG & $12.07 \pm 2.39$ & $8.02-14.61$ \\
Non-grafted+MSCs & $11.42 \pm 2.35$ & $6.37-13.45$ \\
\hline
\end{tabular}

SD: Standard deviation; FGF: Fibroblast growth factor; TIG: Tigecycline; MSCs: Mesenchymal stem cells; PTFE; Polytetrafluoroethylene; TGF- $\beta 1$ : Transforming growth factor-beta 1; IL-1 $\alpha$ : Interleukin 1 alpha; VEGF: Vascular endothelial growth factor; TNF- $\alpha$ : Tumor necrosis factor alpha; PDGF: Platelet-derived growth factor; CASP3: Caspase 3.

with MSCs, were evaluated in terms of colony counts. Mesenchymal stem cell treatment was more successful in groups 6 and 8 than in group $4(\mathrm{p}<0.001)$. Treatment successes in PTFE groups and non-grafted groups were similar ( $p>0.05)$. The overall success of the treatments was assessed by the number of rats with MRSE recurrence, regardless of graft use. The difference between untreated group 2 and tigecycline groups and MSCs groups was statistically significant $(\mathrm{p}=0.016$, $\mathrm{p}=0.016$ ). There was no significant difference between group 2 and Dacron groups in terms of reproductive status. The difference between Dacron groups and PTFE groups and non-grafted groups was significant $(\mathrm{p}=0.047, \mathrm{p}=0.047)$. The results were similar in PTFE groups and non-grafted groups 7 and $8(\mathrm{p}>0.05)$.

Enzyme-linked immunosorbent assay results of all groups of FGF, TGF- $\beta 1$, IL- $1 \alpha$, VEGF, TNF- $\alpha$, PDGF and CASP3 are shown in Table III. KolmogorovSmirnov test showed that all variables had a normal distribution. Descriptive statistical analysis results are given in Table 3 . Because the variances were homogeneous, multiple comparisons between groups were performed using the lysergic acid diethylamide (LSD) method (Tables IV, V). Variables that differ between groups are given in Table 5. ANOVA analysis was performed because the variables had a normal distribution, and significant differences were found between the groups in the IL- $1 \alpha$, VEGF and CASP3 variables. Multiple post-hoc tests were performed to identify the different groups (Table VI).

\section{DISCUSSION}

The results of this study can be summarized in two statements. Firstly, it was shown that both MSCs implantation and tigecycline treatment are effective against MRSE at vascular graft site and soft 
TABLE IV

Test of homogeneity of variances

\begin{tabular}{lcccc}
\hline & Levene statistic & df1 & df2 & $p$ \\
\hline Fibroblast growth factor & 2.759 & 7 & 48 & 0.02 \\
Transforming growth factor-beta 1 & 2.646 & 7 & 48 & 0.02 \\
Interleukin 1 alpha & 1.897 & 7 & 48 & 0.09 \\
Vascular endothelial growth factor & 0.792 & 7 & 48 & 0.60 \\
Tumor necrosis factor alpha & 2.668 & 7 & 48 & 0.02 \\
Platelet-derived growth factor & 2.321 & 7 & 48 & 0.04 \\
Caspase 3 & 1.906 & 7 & 48 & 0.09 \\
\hline
\end{tabular}

df: Degree of freedom.

tissue infections. Secondly, these two anti-infective treatments are successful in the treatment of MRSE infections at PTFE grafted sites where they fail to control and treat bacterial colonization at Dacron graft site infections.

Vascular graft infection is a rare complication after graft implantation but is associated with a high mortality rate (up to $75 \%$ ) and a high rate of major amputation (as high as 70\%). ${ }^{[4,33,34]}$ For particularly open lower extremity injury and fractures, both late diagnosis ${ }^{[6]}$ and late revascularization due to graft problems including thrombosis and infections increase the amputation rates. Thus, for these clinical scenarios, more aggressive and rapid treatment has to be developed.

TABLE V

Results of enzyme-linked immunosorbent assay tests

\begin{tabular}{|c|c|c|c|c|c|}
\hline & Sum of Squares & df & Mean square & $\mathrm{F}$ & $p$ \\
\hline \multicolumn{6}{|c|}{ Fibroblast growth factor } \\
\hline Between groups & 90541.80 & 7 & 12934.54 & 1.50 & 0.19 \\
\hline Within groups & 411570.77 & 48 & 8574.39 & & \\
\hline Total & 502112.57 & 55 & & & \\
\hline \multicolumn{6}{|c|}{ Transforming growth factor beta-1 } \\
\hline Between groups & 5848.20 & 7 & 835.45 & 0.83 & 0.56 \\
\hline Within groups & 47963.59 & 48 & 999.24 & & \\
\hline Total & 53811.79 & 55 & & & \\
\hline \multicolumn{6}{|l|}{ Interleukin 1 alpha } \\
\hline Between groups & 73264.58 & 7 & 10466.37 & 4.73 & 0.00 \\
\hline Within groups & 106222.72 & 48 & 2212.97 & & \\
\hline Total & 179487.31 & 55 & & & \\
\hline \multicolumn{6}{|c|}{ Vascular endothelial growth factor } \\
\hline Between groups & 350022.78 & 7 & 50003.25 & 4.63 & 0.001 \\
\hline Within groups & 517929.61 & 48 & 10790.20 & & \\
\hline Total & 867952.39 & 55 & & & \\
\hline \multicolumn{6}{|c|}{ Tumor necrosis factor alpha } \\
\hline Between groups & 116724.61 & 7 & 16674.94 & 1.30 & 0.27 \\
\hline Within groups & 615329.49 & 48 & 12819.36 & & \\
\hline Total & 732054.10 & 55 & & & \\
\hline \multicolumn{6}{|c|}{ Platelet-derived growth factor } \\
\hline Between groups & 11.97 & 7 & 1.71 & 1.47 & 0.20 \\
\hline Within groups & 55.59 & 48 & 1.15 & & \\
\hline Total & 67.57 & 55 & & & \\
\hline \multicolumn{6}{|l|}{ Caspase 3} \\
\hline Between groups & 73.73 & 7 & 10.53 & 3.79 & 0.002 \\
\hline Within groups & 133.13 & 48 & 2.77 & & \\
\hline Total & 206.87 & 55 & & & \\
\hline
\end{tabular}

df: Degree of freedom. 


\section{TABLE VI}

Multiple post-hoc tests

\begin{tabular}{|c|c|c|c|c|c|c|c|}
\hline \multirow[b]{2}{*}{ Group name (I) } & \multirow[b]{2}{*}{ Group name (J) } & \multicolumn{2}{|c|}{$\begin{array}{l}\text { Dependent variable: } \\
\qquad \mathrm{IL}-1 \alpha\end{array}$} & \multicolumn{2}{|c|}{$\begin{array}{c}\text { Dependent variable: } \\
\text { VEGF }\end{array}$} & \multicolumn{2}{|c|}{$\begin{array}{c}\text { Dependent variable: } \\
\text { Caspase-3 }\end{array}$} \\
\hline & & $\begin{array}{c}\text { Mean difference } \\
(\mathrm{I-J)}\end{array}$ & $p$ & $\begin{array}{c}\text { Mean difference } \\
(\mathrm{I}-\mathrm{J})\end{array}$ & $p$ & $\begin{array}{l}\text { Mean difference } \\
(\mathrm{I}-\mathrm{J})\end{array}$ & $p$ \\
\hline \multirow{7}{*}{ Control } & Infected control & $80.38^{*}$ & 0.002 & $-123.11^{*}$ & 0.031 & -1.21 & 0.18 \\
\hline & Dacron+TIG & 41.86 & 0.102 & -42.94 & 0.443 & 1.35 & 0.14 \\
\hline & Dacron+MSCs & 46.86 & 0.068 & $-139.14^{*}$ & 0.016 & -1.50 & 0.10 \\
\hline & PTFE+TIG & 15.94 & 0.529 & $-249.89^{*}$ & 0.000 & $-2.46^{*}$ & 0.01 \\
\hline & PTFE+MSC & -35.37 & 0.166 & -100.72 & 0.076 & $-2.16^{*}$ & 0.02 \\
\hline & Non-grafted+TIG & -19.48 & 0.442 & $-210.66^{*}$ & 0.000 & -1.33 & 0.14 \\
\hline & Non-grafted+MSCs & -7.40 & 0.770 & -56.79 & 0.311 & -.68 & 0.44 \\
\hline \multirow{7}{*}{ Infected control } & Control & $-80.38^{*}$ & 0.002 & $123.11^{*}$ & 0.031 & 1.21 & 0.18 \\
\hline & Dacron+TIG & -38.52 & 0.132 & 80.16 & 0.155 & $2.57^{\star}$ & 0.01 \\
\hline & Dacron+MSCs & -33.52 & 0.189 & -16.03 & 0.774 & -0.29 & 0.75 \\
\hline & PTFE+TIG & $-64.43^{*}$ & 0.014 & $-126.77^{*}$ & 0.027 & -1.24 & 0.17 \\
\hline & PTFE+MSC & $-115.76^{\star}$ & 0.000 & 22.39 & 0.689 & -0.94 & 0.29 \\
\hline & Non-grafted+TIG & $-99.87^{*}$ & 0.000 & -87.55 & 0.121 & -0.12 & 0.89 \\
\hline & Non-grafted+MSCs & $-87.79^{*}$ & 0.001 & 66.31 & 0.238 & 0.52 & 0.56 \\
\hline \multirow{7}{*}{ Dacron+TIG } & Control & -41.86 & 0.102 & 42.94 & 0.443 & -1.35 & 0.14 \\
\hline & Infected control & 38.52 & 0.132 & -80.16 & 0.155 & $-2.57^{*}$ & 0.01 \\
\hline & Dacron+MSCs & 5.00 & 0.843 & -96.20 & 0.090 & $-2.86^{*}$ & 0.002 \\
\hline & PTFE+TIG & -25.91 & 0.308 & $-206.94^{*}$ & 0.001 & $-3.81^{*}$ & 0.00 \\
\hline & PTFE+MSCs & $-77.24^{*}$ & 0.003 & -57.77 & 0.303 & $-3.51^{*}$ & 0.00 \\
\hline & Non-grafted+TIG & $-61.34^{*}$ & 0.018 & $-167.71^{*}$ & 0.004 & $-2.69^{*}$ & 0.004 \\
\hline & Non-grafted+MSCs & -49.26 & 0.056 & -13.84 & 0.804 & $-2.04^{*}$ & 0.03 \\
\hline \multirow{7}{*}{ Dacron+MSCs } & Control & -46.86 & 0.068 & $139.14^{\star}$ & 0.016 & 1.50 & 0.10 \\
\hline & Infected control & 33.52 & 0.189 & 16.03 & 0.774 & 0.29 & 0.75 \\
\hline & Dacron+TIG & -5.00 & 0.843 & 96.20 & 0.090 & $2.86^{*}$ & 0.002 \\
\hline & PTFE+TIG & -30.91 & 0.225 & -110.74 & 0.052 & -0.95 & 0.29 \\
\hline & PTFE+MSCs & $-82.24^{\star}$ & 0.002 & 38.42 & 0.492 & -0.65 & 0.47 \\
\hline & Non-grafted+TIG & $-66.35^{\star}$ & 0.011 & -71.51 & 0.204 & 0.16 & 0.85 \\
\hline & Non-grafted+MSCs & $-54.27^{\star}$ & 0.036 & 82.35 & 0.145 & 0.81 & 0.36 \\
\hline \multirow{7}{*}{ PTFE+TIG } & Control & -15.94 & 0.529 & $249.89^{*}$ & 0.000 & $2.46^{*}$ & 0.01 \\
\hline & Infected control & $64.43^{*}$ & 0.014 & $126.77^{*}$ & 0.027 & 1.24 & 0.17 \\
\hline & Dacron+TIG & 25.91 & 0.308 & $206.94^{*}$ & 0.001 & $3.81^{*}$ & 0.00 \\
\hline & Dacron+MSCs & 30.91 & 0.225 & 110.74 & 0.052 & 0.95 & 0.29 \\
\hline & PTFE+MSCs & $-51.32^{*}$ & 0.047 & $149.16^{*}$ & 0.010 & 0.30 & 0.74 \\
\hline & Non-grafted+TIG & -35.43 & 0.165 & 39.22 & 0.483 & 1.12 & 0.21 \\
\hline & Non-grafted+MSCs & -23.35 & .358 & $193.09^{*}$ & .001 & 1.77 & 0.05 \\
\hline
\end{tabular}


TABLE VI

Continued

\begin{tabular}{|c|c|c|c|c|c|c|c|}
\hline \multirow[b]{2}{*}{ Group name (I) } & \multirow[b]{2}{*}{ Group name (J) } & \multicolumn{2}{|c|}{$\begin{array}{l}\text { Dependent variable: } \\
\qquad \mathrm{IL}-1 \alpha\end{array}$} & \multicolumn{2}{|c|}{$\begin{array}{c}\text { Dependent variable: } \\
\text { VEGF }\end{array}$} & \multicolumn{2}{|c|}{$\begin{array}{l}\text { Dependent variable: } \\
\text { Caspase-3 }\end{array}$} \\
\hline & & $\begin{array}{c}\text { Mean difference } \\
(I-J)\end{array}$ & $p$ & $\begin{array}{c}\text { Mean difference } \\
(I-J)\end{array}$ & $p$ & $\begin{array}{l}\text { Mean differ- } \\
\text { ence }(I-J)\end{array}$ & $p$ \\
\hline \multirow{7}{*}{ PTFE+MSCs } & Control & 35.37 & 0.166 & 100.72 & 0.076 & $2.16^{\star}$ & 0.02 \\
\hline & Infected control & $115.76^{*}$ & 0.000 & -22.39 & 0.689 & 0.94 & 0.29 \\
\hline & Dacron+TIG & $77.24^{*}$ & 0.003 & 57.77 & 0.303 & $3.51^{*}$ & 0.00 \\
\hline & Dacron+MSCs & $82.24^{*}$ & 0.002 & -38.42 & 0.492 & 0.65 & 0.47 \\
\hline & PTFE+TIG & $51.32^{*}$ & 0.047 & $-149.16^{*}$ & 0.010 & -0.30 & 0.74 \\
\hline & Non-grafted+TIG & 15.89 & 0.530 & -109.94 & 0.053 & 0.82 & 0.36 \\
\hline & Non-grafted+MSCs & 27.97 & 0.271 & 43.92 & 0.433 & 1.47 & 0.11 \\
\hline \multirow{7}{*}{ Non-Grafted+TIG } & Control & 19.48 & 0.442 & $210.66^{*}$ & 0.000 & 1.33 & 0.14 \\
\hline & Infected control & $99.87^{*}$ & 0.000 & 87.55 & 0.121 & 0.12 & 0.89 \\
\hline & Dacron+TIG & $61.34^{*}$ & 0.018 & $167.71^{*}$ & 0.004 & $2.69^{*}$ & 0.004 \\
\hline & Dacron+MSCs & $66.35^{\star}$ & 0.011 & 71.51 & 0.204 & -0.16 & 0.85 \\
\hline & PTFE+TIG & 35.43 & 0.165 & -39.22 & 0.483 & -1.12 & 0.21 \\
\hline & PTFE+MSCs & -15.89 & 0.530 & 109.94 & 0.053 & -0.82 & 0.36 \\
\hline & Non-grafted+MSCs & 12.08 & 0.633 & $153.87^{*}$ & 0.008 & 0.65 & 0.47 \\
\hline \multirow{7}{*}{ Non-Grafted+MSCs } & Control & 7.40 & 0.770 & 56.79 & 0.311 & 0.68 & 0.44 \\
\hline & Infected control & $87.79^{\star}$ & 0.001 & -66.31 & 0.238 & -0.52 & 0.56 \\
\hline & Dacron+TIG & 49.26 & 0.056 & 13.84 & 0.804 & $2.04^{*}$ & 0.03 \\
\hline & Dacron+MSCs & $54.27^{\star}$ & 0.036 & -82.35 & 0.145 & -0.81 & 0.36 \\
\hline & PTFE+TIG & 23.35 & 0.358 & $-193.09^{*}$ & 0.001 & -1.77 & 0.05 \\
\hline & PTFE+MSCs & -27.97 & 0.271 & -43.92 & 0.433 & -1.47 & 0.11 \\
\hline & Non-grafted+TIG & -12.0800 & 0.633 & $-153.87^{\star}$ & 0.008 & -0.65 & 0.47 \\
\hline
\end{tabular}

IL-1 $\alpha$ : Interleukin 1 alpha; VEGF: Vascular endothelial growth factor; TIG: Tigecycline; MSCs: Mesenchymal stem cells; PTFE: Polytetrafluoroethylene; ${ }^{*}$ Mean difference is significant at 0.05 level.

Staphylococcus aureus, Staphylococcus epidermidis and gram-negative Escherichia coli (E. coli) are the most common pathogens isolated in vascular graft infections. The development of resistance and reduction of susceptibility ${ }^{[35]}$ to methicillin at the beginning and vancomycin later by the staphylococci gave rise to investigations for alternative treatment options for vascular graft infections. ${ }^{[10]}$ Tigecycline, the first generation of glycycline antibiotics, is one of the choices of treatment in infections caused by methicillin-resistant Staphylococcus aureus (MRSA), MRSE, and vancomycin-resistant enterococcus infections. ${ }^{[36,37]}$ Thus, it is one of the alternative antimicrobial treatments for vascular graft infections resistant to methicillin or vancomycin. Although it is out of the aim of this study, we demonstrated the effective antimicrobial efficacy of tigecycline for both PTFE grafted and non-grafted MRSE infections. However, there are some concerns about tigecycline treatment. A meta-analysis performed by Shen et al. ${ }^{[38]}$ has reported lower cure rates with tigecycline according to data extracted from 14 studies evaluating totally 5,663 patients. In the same meta-analysis, although not statistically significant, reported microbiological treatment success was numerically lower than compared treatment regimens including MRSA, MRSE, E. coli, and Klebsiella. The most important and remarkable negative finding of tigecycline treatment is the significantly higher number of side effects particularly in the digestive, hematopoietic and lymphatic systems. ${ }^{[37]}$ Also, numerically higher mortality is observed although statistically not significant. ${ }^{[37,38]}$ 
Mesenchymal stem cells modulate the activity of macrophages. These effects have been demonstrated in $e x$-vivo demonstration by macrophages induced by Toll-like receptor ligands such as lipopolysaccharide, zymosan or polyinosinic-polycytidylic acid. ${ }^{[39]}$ When macrophages are stimulated with bacterial or viral agents, they secrete inflammatory factors such as TNF- $\alpha$, IL-1, and IL-6. ${ }^{[13]}$ In our study, successful results with MSCs similar to tigecycline treatment were obtained in terms of bacterial colonization in both PTFE grafted and non-grafted infection sites. The antibacterial properties of MSCs are related with their immunomodulating and direct antibacterial effect on the infectious agent. ${ }^{[13,15,19,40]}$ As immunomodulators, MSCs initiate a shift in the ratio of $\mathrm{T}$ helper cells through $\mathrm{T}$ helper 2 anti-inflammatory subtype and increase differentiation of naive $\mathrm{T}$ cells to regulatory phenotype. ${ }^{[13]}$ They also modulate the activity of macrophages. The direct anti-infectious effects are provided by secretion of antibacterial peptides and intensification of phagocytosis. ${ }^{[1,19]}$ The increase in IL- 1 and particularly its $\alpha$ subtype may be responsible from anti-infective properties of MSCs because it is well documented that IL- $1 \alpha$ promotes the release of chemokines and adhesion molecules by inducing endothelial cells. ${ }^{[11,42]}$ Thus, it provides white blood cells to reach the infected area with chemotaxis and augments inflammation. In our study, MSCs implantation increased IL-1 $\alpha$ levels, particularly in the PTFE groups more than other groups. Bartosh et al. ${ }^{[43]}$ also demonstrated that MSCs triggered IL-1 signaling and secretion of inflammation and immune modulators. In the literature, there are studies supporting these effects of experimental MSCs, many of which were performed on sepsis models. ${ }^{[19]}$ Devaney et al. ${ }^{[18]}$ demonstrated in a mouse model of $E$. coli-induced pneumonia a lesser intensity of lung damage, lower bacterial load, and higher intensity of phagocytosis following the intratracheal administration of MSCs. Also, Pedrazza et al. ${ }^{[21]}$ showed a significant decrease in mortality in the group that received MSCs compared to the control group which was a mouse model of sepsis induced by the administration of $E$. coli into the peritoneal cavity. These reported results are parallel to those obtained for vascular graft site infections in our study.

Another remarkable finding of this study is the resistance of MRSE infections to both MSCs implantation and tigecycline treatment. This significant difference in Dacron grafted groups can be considered to be indicative of the susceptibility of the Dacron grafts to infection. Schmitt et al. ${ }^{[4]}$ demonstrated that bacterial adherence to knitted
Dacron grafts is more than adherence to PTFE grafts, which is related to the surface area, porosity, and chemical structure. The difference in these structural characteristics creates the difference in bacterial affinity between the PTFE and Dacron graft materials. In addition, they underlined that PTFE is more hydrophobic than Dacron resulting in forming a lesser number of bonds with hydrophobic cell walls. This predisposition of Dacron graft to infection needs to be demonstrated by further histopathological investigations. However, we advise preferring PTFE grafts for vascular grafting particularly for repairing vascular injury at the open wound or fracture sites.

This experimental study has some limitations. Our invivo model used a direct method of MRSE colonization on the graft. Thus, grafts are not comparable to the animal model for applying into a blood vessel. The antibiotics were administered intraperitoneally instead of intravenously and antibiotic binding to the grafts was not assessed. Additionally, the effect of the combination of antibiotics with MSCs implantation was not demonstrated in a group. All the limitations mentioned above are inherent to experimental studies; therefore, further human studies are needed. MSCs practice is not yet cheaper than costly antibiotics; however, we may assume that the cost will decrease as this practice becomes more widespread.

In conclusion, MSCs can be a good alternative treatment option as single or combination therapy for vascular graft infections. They can be an effective part of treatment for patients with organ failures where sensitive dose adjustment for antibiotics is required, for patients with a high risk of graft infection due to contamination of vascular injury site, and also for those under 18 years of age for whom many antibiotics cannot be used due to side effects. In addition, for potential resistance of MRSE to tigecycline in near future, MSC therapy alone or combination with antibiotics may be a good alternative treatment choice. Moreover, MSC therapy may slow down and delay the time course of resistance development to tigecycline therapy. As we mentioned, MSC culture techniques are not cheaper yet; however, we believe that MSCs will be cultured at lower-cost techniques in local laboratories in near future. We need further animal and human studies to find out the efficacy of MSC treatment in infected vascular grafts.

\section{Acknowledgments}

We thank Prof. Dr. Sevgi Yurt Öncel for supporting the statistical analyses of this manuscript.

\section{Declaration of conflicting interests}

The authors declared no conflicts of interest with respect to the authorship and/or publication of this article. 


\section{Funding}

This study was supported by Kırıkkale University Scientific Research Council.

\section{REFERENCES}

1. Caudle RJ, Stern PJ. Severe open fractures of the tibia. J Bone Joint Surg [Am] 1987;69:801-7.

2. Howe HR Jr, Poole GV Jr, Hansen KJ, Clark T, Plonk GW, Koman LA, et al. alvage of lower extremities following combined orthopedic and vascular trauma. A predictive salvage index. Am Surg 1987;53:205-8.

3. Frisvoll C, Clarke-Jenssen J, Madsen JE, Flugsrud G, Frihagen F, Andreassen GS, et al. Long-term outcomes after high-energy open tibial fractures: Is a salvaged limb superior to prosthesis in terms of physical function and quality of life? Eur J Orthop Surg Traumatol 2019;29:899-906.

4. Legout L, D'Elia PV, Sarraz-Bournet B, Haulon S, Meybeck A, Senneville E, et al. Diagnosis and management of prosthetic vascular graft infections. Med Mal Infect. 2012;42:102-9.

5. Erb S1, Sidler JA1, Elzi L1, Gurke L2, Battegay M1, Widmer $\mathrm{AF}$, et al. Surgical and antimicrobial treatment of prosthetic vascular graft infections at different surgical sites: a retrospective study of treatment outcomes. PLoS One 2014;9:e112947.

6. Halvorson JJ, Anz A, Langfitt M, Deonanan JK, Scott A, Teasdall RD, et al. Vascular injury associated with extremity trauma: initial diagnosis and management. J Am Acad Orthop Surg 2011;19:495-504.

7. Herscu G, Wilson SE. Prosthetic infection: lessons from treatment of the infected vascular graft. Surg Clin North Am 2009;89:391-401

8. Darouiche RO. Treatment of infections associated with surgical implants. N Engl J Med 2004;350:1422-9

9. Bergamini TM, Corpus RA Jr, Brittian KR, Peyton JC, Cheadle WG. The natural history of bacterial biofilm graft infection. J Surg Res 1994;56:393-6.

10. Sacar S, Sacar M, Kaleli I, Toprak S, Cevahir N, Teke $\mathrm{Z}$, et al. Linezolid compared with vancomycin for the prevention of methicillin-resistant Staphylococcus aureus or Staphylococcus epidermidis vascular graft infection in rats: A randomized, controlled, experimental study. Curr Ther Res Clin Exp 2007;68:23-31.

11. Elens M, Dusoruth M, Astarci P, Mastrobuoni S, Bosiers MJ, Nardella J, et al. Management and Outcome of Prosthetic Vascular Graft Infections: A Single Center Experience. Vasc Endovascular Surg 2018;52:181-87.

12. Corcione A, Benvenuto F, Ferretti E, Giunti D, Cappiello V, Cazzanti F, et al. Human mesenchymal stem cells modulate B-cell functions. Blood 2006;107:367-72.

13. Spees JL, Lee RH, Gregory CA. Mechanisms of mesenchymal stem/stromal cell function. Stem Cell Res Ther 2016;7:125.

14. Matthay MA, Pati S, Lee JW. Concise review: Mesenchymal stem (Stromal) cells: Biology and preclinical evidence for therapeutic potential for organ dysfunction following trauma or sepsis. Stem Cells 2017;35:316-324.

15. Lee RH, Pulin AA, Seo MJ, Kota DJ, Ylostalo J, Larson BL, et al. Intravenous hMSCs improve myocardial infarction in mice because cells embolized in lung are activated to secrete the anti-inflammatory protein TSG-6. Cell Stem Cell 2009;5:54-63.

16. Kota DJ, Wiggins LL, Yoon N, Lee RH. TSG-6 produced by hMSCs delays the onset of autoimmune diabetes by suppressing Th1 development and enhancing tolerogenicity. Diabetes 2013;62:2048-58.

17. Baron F, Storb R. Mesenchymal stromal cells: a new tool against graft-versus-host disease? Biol Blood Marrow Transplant 2012;18:822-40.

18. Devaney J, Horie S, Masterson C, Elliman S, Barry F, O’Brien $\mathrm{T}$, et al. Human mesenchymal stromal cells decrease the severity of acute lung injury induced by E. coli in the rat. Thorax 2015;70:625-35.

19. Horák J, Nalos L, Martínková V, Beneš J, Štengl M, Matějovič M. Mesenchymal stem cells in sepsis and associated organ dysfunction: A promising future or blind alley? Stem Cells Int 2017;2017:7304121.

20. Asano K, Yoshimura S, Nakane A. Adipose tissuederived mesenchymal stem cells attenuate staphylococcal enterotoxin a-induced toxic shock. Infect Immun 2015;83:3490-6.

21. Pedrazza L, Lunardelli A, Luft C, Cruz CU, de Mesquita FC, Bitencourt S, et al. Mesenchymal stem cells decrease splenocytes apoptosis in a sepsis experimental model. Inflamm Res 2014;63:719-28.

22. Eren Y, Adanır O, Dinçel YM, Genç E, Arslan YZ, Çağlar A. Effects of low molecular weight heparin and rivaroxaban on rat Achilles tendon healing. Eklem Hastalik Cerrahisi 2018;29:13-9.

23. Seguin JC, Walker RD, Caron JP, Kloos WE, George CG, Hollis RJ, et al. Methicillin-resistant Staphylococcus aureus outbreak in a veterinary teaching hospital: potential human-to-animal transmission. J Clin Microbiol 1999;37:1459-63.

24. Goessens WH, Mouton JW, Ten Kate MT, Sörgel F, Kinzig M, Bakker-Woudenberg IA. The therapeutic effect of tigecycline, unlike that of Ceftazidime, is not influenced by whether the Klebsiella pneumoniae strain produces extendedspectrum beta-lactamases in experimental pneumonia in rats. Antimicrob Agents Chemother 2013;57:643-6.

25. Schneider S, Unger M, van Griensven M, Balmayor ER. Adipose-derived mesenchymal stem cells from liposuction and resected fat are feasible sources for regenerative medicine. Eur J Med Res 2017;22:17.

26. Mushahary D, Spittler A, Kasper C, Weber V, Charwat $\mathrm{V}$. Isolation, cultivation, and characterization of human mesenchymal stem cells. Cytometry A 2018;93:19-31.

27. Mori Y, Ohshimo J, Shimazu T, He H, Takahashi A, Yamamoto $\mathrm{Y}$, et al. Improved explant method to isolate umbilical cord-derived mesenchymal stem cells and their immunosuppressive properties. Tissue Eng Part C Methods 2015;21:367-72.

28. Talaei-Khozani T, Borhani-Haghighi M, Ayatollahi M, Vojdani Z. An in vitro model for hepatocyte-like cell differentiation from Wharton's jelly derived-mesenchymal stem cells by cell-base aggregates. Gastroenterol Hepatol Bed Bench 2015;8:188-99.

29. Hernández-Richter T, Schardey HM, Wittmann F, Mayr S, Schmitt-Sody M, Blasenbreu S, et al. Rifampin and Triclosan but not silver is effective in preventing bacterial infection of vascular dacron graft material. Eur J Vasc Endovasc Surg 2003;26:550-7. 
30. Takahashi H, Sakata N, Yoshimatsu G, Hasegawa S, Kodama S. Regenerative and Transplantation Medicine: Cellular Therapy Using Adipose Tissue-Derived Mesenchymal Stromal Cells for Type 1 Diabetes Mellitus. J Clin Med 2019;8. pii: E249.

31. Sarıkaya B, Yumuşak N, Yigin A, Sipahioğlu S, Yavuz Ü, Altay MA. Comparison of the effects of human recombinant epidermal growth factor and platelet-rich plasma on healing of rabbit patellar tendon. Eklem Hastalik Cerrahisi 2017;28:92-9.

32. Chiswick EL, Duffy E, Japp B, Remick D. Detection and quantification of cytokines and other biomarkers. Methods Mol Biol 2012;844:15-30.

33. O'Connor S, Andrew P, Batt M, Becquemin JP. A systematic review and meta-analysis of treatments for aortic graft infection. J Vasc Surg 2006;44:38-45.

34. Legout L, Sarraz-Bournet B, D'Elia PV, Devos P, Pasquet A, Caillaux $M$, et al. Characteristics and prognosis in patients with prosthetic vascular graft infection: a prospective observational cohort study. Clin Microbiol Infect 2012;18:352-8.

35. Lee JYH, Monk IR, Gonçalves da Silva A, Seemann T, Chua KYL, Kearns A, et al. Global spread of three multidrugresistant lineages of Staphylococcus epidermidis. Nat Microbiol 2018;3:1175-85.

36. Bhattacharya M, Parakh A, Narang M. Tigecycline. J Postgrad Med 2009;55:65-8.

37. Cai $Y$, Li R, Liang B, Bai N, Liu $Y$, Wang R. In vitro antimicrobial activity and mutant prevention concentration of colistin against Acinetobacter baumannii. Antimicrob Agents Chemother 2010;54:3998-9.

38. Shen F, Han Q, Xie D, Fang M, Zeng H, Deng Y. Efficacy and safety of tigecycline for the treatment of severe infectious diseases: an updated meta-analysis of RCTs. Int J Infect Dis 2015;39:25-33.

39. Moresco EM, LaVine D, Beutler B. Toll-like receptors. Curr Biol 2011;21:R488-93.

40. Németh K, Leelahavanichkul A, Yuen PS, Mayer B, Parmelee A, Doi $\mathrm{K}$, et al. Bone marrow stromal cells attenuate sepsis via prostaglandin $\mathrm{E}(2)$-dependent reprogramming of host macrophages to increase their interleukin-10 production. Nat Med 2009;15:42-9.

41. Furie MB, Randolph GJ. Chemokines and tissue injury. Am J Pathol 1995;146:1287-301.

42. Kaneider NC, Leger AJ, Kuliopulos A. Therapeutic targeting of molecules involved in leukocyte-endothelial cell interactions. FEBS J 2006;273:4416-24.

43. Bartosh TJ, Ylöstalo JH, Bazhanov N, Kuhlman J, Prockop DJ. Dynamic compaction of human mesenchymal stem/ precursor cells into spheres self-activates caspasedependent IL1 signaling to enhance secretion of modulators of inflammation and immunity (PGE2, TSG6, and STC1). Stem Cells 2013;31:2443-56.

44. Schmitt DD, Bandyk DF, Pequet AJ, Towne JB. Bacterial adherence to vascular prostheses. A determinant of graft infectivity. J Vasc Surg 1986;3:732-40. 